\title{
Concomitant pancreatic endocrine neoplasm and intraductal papillary mucinous neoplasm: a case report and literature review
}

\author{
Yoshie Kadota', Masahiro Shinoda1*, Minoru Tanabe ${ }^{1}$, Hanako Tsujikawa ${ }^{2}$, Akihisa Ueno $^{3}$, Yohei Masugi²,

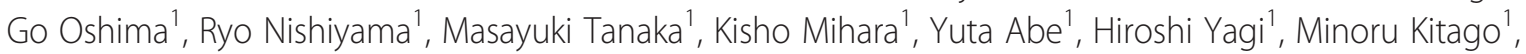 \\ Osamu Itano', Shigeyuki Kawachi ${ }^{1}$, Koichi Aiura ${ }^{4}$, Akihiro Tanimoto ${ }^{3}$, Michiie Sakamaoto ${ }^{2}$ and Yuko Kitagawa ${ }^{1}$
}

\begin{abstract}
We report a case of concomitant pancreatic endocrine neoplasm (PEN) and intraductal papillary mucinous neoplasm (IPMN). A 74-year-old man had been followed-up for mixed-type IPMN for 10 years. Recent magnetic resonance images revealed an increase in size of the branch duct IPMN in the pancreas head, while the dilation of the main pancreatic duct showed minimal change. Although contrast-enhanced computed tomography and magnetic resonance imaging did not reveal any nodules in the branch duct IPMN, endoscopic ultrasound indicated a suspected nodule in the IPMN. A malignancy in the branch duct IPMN was suspected and we performed pyloruspreserving pancreatoduodenectomy with lymphadenectomy. The resected specimen contained a cystic lesion, $10 \mathrm{x}$ $10 \mathrm{~mm}$ in diameter, in the head of the pancreas. Histological examination revealed that the dilated main pancreatic duct and the branch ducts were composed of intraductal papillary mucinous adenoma with mild atypia. No evidence of carcinoma was detected in the specimen. Incidentally, a 3-mm nodule consisting of small neuroendocrine cells was found in the main pancreatic duct. The cells demonstrated positive staining for chromogranin A, synaptophysin, and glucagon but negative staining for insulin and somatostatin. Therefore, the 3$\mathrm{mm}$ nodule was diagnosed as a PEN. Since the mitotic count per 10 high-power fields was less than 2 and the Ki67 index was less than 2\%, the PEN was pathologically classified as low-grade (G1) according to the 2010 World Health Organization (WHO) criteria. Herein, we review the case and relevant studies in the literature and discuss issues related to the synchronous occurrence of the relatively rare tumors, PEN and IPMN.
\end{abstract}

Keywords: Pancreatic endocrine neoplasm, Intraductal papillary mucinous neoplasm

\section{Background}

Pancreatic endocrine neoplasm (PEN) and intraductal papillary neoplasm (IPMN) are both relatively rare tumors among the primary pancreatic neoplasms, with reported frequencies of approximately 0.4 per 100,000 and 1 per 100,000 , respectively $[1,2]$. There have been only a few published reports of cases in which PEN and IPMN were present concomitantly [3-10]. We encountered a rare case who underwent pancreatoduodenectomy for pancreas head IPMN and whose postoperative pathological examination revealed the existence of concomitant PEN. We present

\footnotetext{
* Correspondence: masa02114@yahoo.co.jp

'Department of Surgery, School of Medicine, 35 Shinanomachi, Shinjuku, Tokyo 160-8582, Japan

Full list of author information is available at the end of the article
}

this case and discuss issues related to the prevalence and tumorigenesis based on a review of published literature.

\section{Case presentation}

A 74-year-old man was admitted to our hospital for the examination of IPMN in the pancreas head. A cystic lesion, $6 \mathrm{~mm}$ in diameter, in the pancreas head was initially revealed when he underwent ultrasonography for a routine checkup for fatty liver 10 years prior. Magnetic resonance imaging revealed the cystic lesion and a slightly dilated main pancreas duct $(6 \mathrm{~mm})$. The patient was diagnosed with mixed-type (both main and branch pancreatic ducts involved) IPMN and has been carefully followed-up by magnetic resonance imaging every six months for the last 10 years. The most recent magnetic 
resonance images revealed an increase, relative to the second most recent images, in the size of the IPMN (that is, an increase from $8 \mathrm{~mm}$ to $12 \mathrm{~mm}$ ) in the pancreatic head, while the dilation $(6 \mathrm{~mm})$ of the main pancreatic duct showed minimal change (axial image in Figure $1 \mathrm{~A}$ and cholangiopancreatography in Figure 1B). The findings in the branch duct IPMN showed discrepant results with different imaging modalities. While contrastenhanced computed tomography (Figure 1C) and magnetic resonance imaging did not show any nodules in the IPMN, endoscopic ultrasound revealed a suspected nodule $(6 \mathrm{~mm})$. The tumor markers carcinoembryonic antigen (CEA), carbohydrate antigen 19-9 (CA19-9), and DUPAN-2 were all within normal limits. Because there was an increase in the size of the branch duct IPMN, and a preoperative endoscopic ultrasound suggested the existence of a nodule in the IPMN, a malignancy in the branch duct IPMN was suspected and we performed pylorus-preserving pancreatoduodenectomy (PpPD) with lymphadenectomy for diagnostic and therapeutic purposes. The postoperative course was uneventful and the patient was discharged on post-operative Day 29 and has been alive for 18 months.

In the resected specimen, a dilated main pancreas duct, $6 \mathrm{~mm}$ in size, and a cluster of multiple dilated branch pancreas ducts were seen (Figure 2A). Histological examination revealed that both the main and branch pancreas ducts were composed of intraductal papillary mucinous adenoma with mild atypia (Figure $2 \mathrm{~B}, \mathrm{C}$ ). Therefore, we diagnosed this patient as having mixed-type IPMN. Incidentally, a 3-mm nodule of endocrine cells was found in the IPMN lesion in the main pancreas duct (Figure 2B, D). Since the cells demonstrated positive staining for chromogranin A and synaptophysin (Figure 2E, F), the nodule was diagnosed as a PEN. The cells also showed positive staining for glucagon (Figure 2G), and negative staining for insulin and somatostatin. The plasma levels of glucagon, insulin and somatostatin had not been examined preoperatively. Since the mitotic count per 10 high-power fields was less than 2 and the Ki-67 index was less than 2\% (Figure $2 \mathrm{H}$ ), the PEN was pathologically classified as a well-differentiated neuroendocrine tumor and was lowgrade (G1) according to the 2000 and 2010 WHO criteria, respectively $[11,12]$. We retrospectively assessed the preoperative images but did not find a nodule corresponding to the PEN lesion. Regarding the nodule in the branch duct IPMN, we also retrospectively assessed the preoperative images and resected specimen but did not find evidence of a nodule in the cluster of multiple dilated branch pancreas ducts corresponding to the endoscopic ultrasound finding. Thus, we recognize that such nodules can be misdiagnosed or over-diagnosed (that is, generate a false positive result) by endoscopic ultrasound.

\section{Discussion}

Neuroendocrine tumors are neoplasms that arise from cells of the endocrine and nervous systems. They can originate within the pancreas and are quite distinct from the usual form of pancreatic cancer, which arises in the exocrine pancreas. IPMN is a type of neoplasm that grows within the pancreatic ducts and is characterized by the production of thick mucinous fluid. These two disease entities have been thought to be distinct and no tumorigenetic associations between them have been reported in the past. Therefore, the incidence of coexistence of these rare neoplasms should be extremely low if their individual incidences are simply multiplied. However, Marrache et al. [4] reported that the prevalence of association between these tumors was $2.8 \%$ at their institute

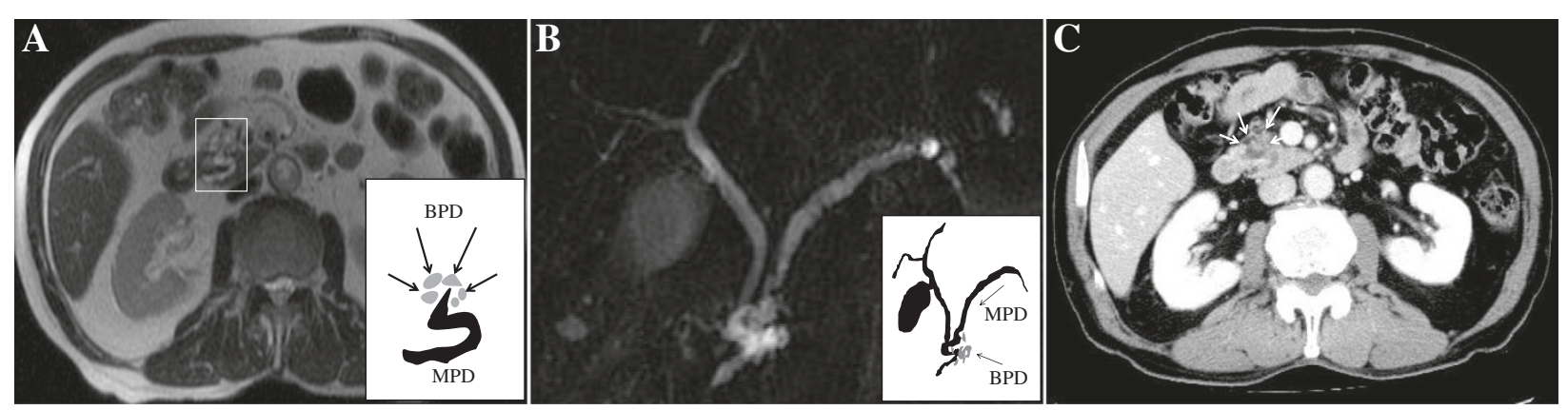

Figure 1 Preoperative imaging diagnoses. A) Magnetic resonance imaging findings (axial, T2-weighted image). The image indicated by a rectangle is illustrated as an inset in the lower right. A black curved line in the illustration indicates the main pancreas duct (MPD). A cystic lesion, illustrated as a cluster of gray areas, is seen around the main pancreas duct. This lesion is a cluster of multiple dilated branch pancreas ducts (BPD) and has been diagnosed as branch intraductal papillary mucinous neoplasm. B) Magnetic resonance cholangiopancreatography findings The image is illustrated as an inset in the lower right. Black lines in the illustration indicate the main pancreas duct (MPD), the biliary tree and the gallbladder. The main pancreatic duct is dilated $(7 \mathrm{~mm})$ and also is markedly curved like an S-shape in the pancreas head. A lesion consisting of multiple dilated branch pancreas ducts (BPD), illustrated as a cluster of gray areas, is seen in the pancreas head. C) Contrast-enhanced computed tomography findings in the portal venous phase. A markedly curved main pancreas duct and multiple dilated branch pancreas ducts (indicated by arrows) are seen in the pancreas head. No enhanced nodules are seen in either the main pancreas duct or dilated branch pancreas ducts. 


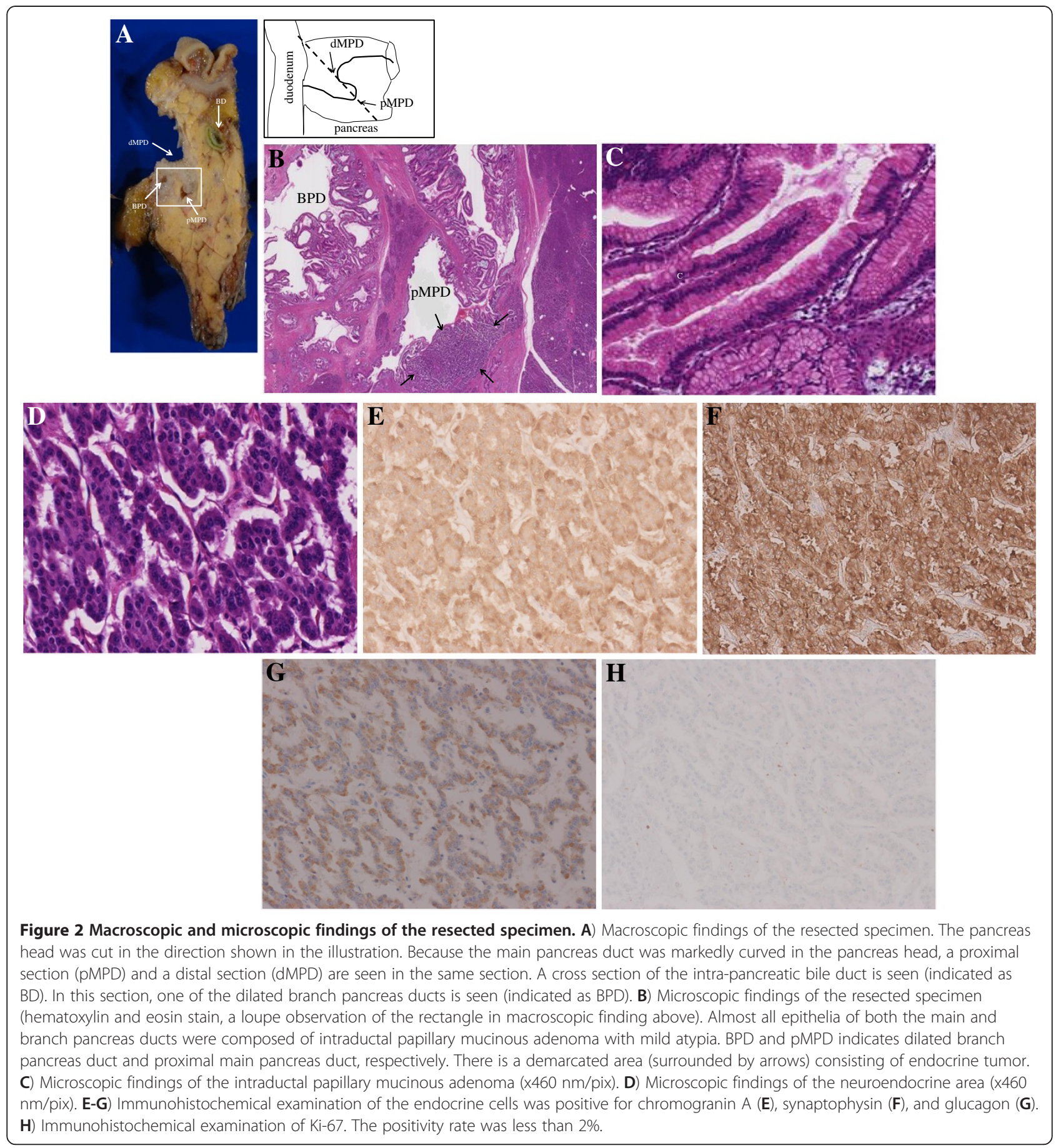

(6 of 211 patients who underwent surgery for a PEN or IPMN). Goh et al. [5] and Gill et al. [10] subsequently reported prevalence rates of 4.6\% (3 of 65 patients who underwent surgery for a PEN or IPMN) and 3.8\% (4 of 104 patients who underwent surgery for IPMN), respectively. These studies suggest that occurrence of PEN and IPMN is more frequent than expected in the past and that potentially concomitant PEN and IPMN may be underdiagnosed. We retrospectively reviewed previous pathological reports of 34 PENs and 40 IPMNs in the past 15 years in our institute, but found no reports of concomitant PEN and IPMN in a total of 74 cases. Given the present case, the incidence of concomitant PEN and IPMN is $1.3 \%$ ( 1 of 75 patients who underwent surgery for PEN or IPMN) in our institute, which seems lower than the rates described above [3-5]. To determine the actual incidence of synchronous occurrence, it will be necessary to examine a large number of patients with PEN or IPMN 
Table 1 Patients with concomitant pancreatic endocrine neoplasm and intraductal papillary mucinous neoplasm reported in the literature

\begin{tabular}{|c|c|c|c|c|c|c|c|c|}
\hline \multirow{2}{*}{$\begin{array}{l}\text { No. } \\
\text { (ref.) }\end{array}$} & \multirow{2}{*}{$\begin{array}{l}\text { Age/ } \\
\text { Sex }\end{array}$} & \multirow{2}{*}{$\begin{array}{l}\text { Preoperative } \\
\text { diagnosis }\end{array}$} & \multirow[t]{2}{*}{ Surgery } & \multicolumn{2}{|r|}{ PEN } & \multicolumn{2}{|c|}{ IPMN } & \multirow{2}{*}{$\begin{array}{l}\text { Postoperative } \\
\text { outcome }\end{array}$} \\
\hline & & & & $\begin{array}{l}\text { Location, size } \\
\quad(\mathrm{mm})\end{array}$ & Pathology1 & $\begin{array}{l}\text { Location, size }(\mathrm{mm}) \text {, } \\
\text { type }\end{array}$ & Pathology2 & \\
\hline 1 (3) & $51 / \mathrm{M}$ & PEN & DP & Tail, 15 & Islet cell tumor with nesidioblastosis & Tail, ND, ND & $\begin{array}{l}\text { IPMH (intraductal papillary } \\
\text { mucinous hyperplasia) }\end{array}$ & ND \\
\hline $2(4)$ & $73 / \mathrm{M}$ & IPMN and PEN & DP & Tail, 28 & Potentially malignant & Tail, ND, Branch & Benign & ND \\
\hline $3(4)$ & 40/F & IPMN and PEN & PD & Head, 11 & Benign & Head, ND, Branch & Benign & ND \\
\hline $4(4)$ & $61 / F$ & IPMN & DP & Tail, 12 & Benign & Tail, ND, Mixed & Borderline & ND \\
\hline $5(4)$ & $55 / F$ & PEN & PD & Head, 30 & $\begin{array}{l}\text { Malignant (duodenal wall invasion, } \\
\text { peripancreatic lymph nodes metastases) }\end{array}$ & Head, ND, Mixed & Benign & ND \\
\hline $6(4)$ & $68 / F$ & IPMN and PEN & $\mathrm{DP}$ & Body, 18 & Benign & Body, ND, Mixed & Benign & ND \\
\hline 7 (4) & $62 / M$ & IPMN & PD & Head, 20 & Potentially malignant & Head, ND, Mixed & Malignant noninvasive & ND \\
\hline $8(5)$ & $65 / F$ & IPMN & TP & Body, 2 & Benign & Head, Body, 40, Mixed & Malignant invasive & $\begin{array}{l}\text { Disease-free, } 10 \\
\text { months }\end{array}$ \\
\hline $9(5)$ & $66 / M$ & IPMN & TP & Tail, 5 & Benign & Entire, 150, Mixed & Malignant invasive & Alive, 70 months \\
\hline $10(5)$ & $58 / \mathrm{M}$ & IPMN & DP & Tail, 8 & Benign & Tail, 18, Branch & Borderline & Alive, 5 months \\
\hline $11(6)$ & $72 / F$ & ND & PD & Head, 25 & PDNC (resional lymph nodes metastases) & Head, ND, ND & Borderline malignant potential & Died, 10 months \\
\hline $12(7)$ & $75 / M$ & IPMN & PD & Head, 35 & WDNC (peripancreatic lymph nodes metastases) & Head, 35, Mixed & $\begin{array}{l}\text { Moderately to poorly } \\
\text { differentiated adenocarcinoma }\end{array}$ & Died, 6 months \\
\hline $13(8)$ & $54 / F$ & ND & PD & Head, ND & ND & Head, 25, Branch & Benign & ND \\
\hline $14(9)$ & $59 / F$ & IPMN and PEN & observation & Body, 7.8 & Benign & Body, Tail, 10 the largest, Branch & ND & Alive, 12 months \\
\hline $15(9)$ & $55 / F$ & IPMN and PEN & enucleation & Head, 20 & Low malignant potential & Head, 5,6,7, Branch & ND & ND \\
\hline $16(10)$ & $67 / M$ & IPMN & TP & Head, 8 & WDNT & Diffuse, 20, Main & Low grade dysplasia & ND \\
\hline $17(10)$ & $72 / F$ & IPMN and PEN & DP & Tail, 16 & WDNC (peripancreatic lymph nodes metastases) & Body, 9, Branch & Low grade dysplasia & ND \\
\hline $18(10)$ & $72 / F$ & IPMN & DP & Body, 9 & WDNT & Body, 15, Branch & Low grade dysplasia & ND \\
\hline $19(10)$ & 76/F & IPMN & TP & Head, 11 & WDNT & Head, 27, Branch & $\begin{array}{l}\text { Well differentiated } \\
\text { adenocarcinoma }\end{array}$ & ND \\
\hline 20 & $74 / \mathrm{M}$ & IPMN & PD & Head, 3 & WDNT/NET G1 & Head, 10, Mixed & Low grade dysplasia & Alive, 12 months \\
\hline
\end{tabular}

1. Pathology of PEN is described according to WHO 2000 criteria, as this was used in all prior studies. As for the present case, the pathology of the PEN is described according to both WHO 2000 and 2010 criteria. 2. The pathology of IPMN is described according to criteria in prior studies [15]. As for the present case, the pathology of IPMN is described according to WHO 2010 criteria [16]

Branch, bhe pancreatoduodenectomy; PDNC, poorly differentiated neuroendocrine carcinoma; PEN, pancreatic endocrine neoplasm; TP, total pancreatectomy; WDNC, well differentiated neuroendocrine carcinoma; WDNT, wellpancreatoduodenectomy; PDNC, poorly 
and carefully examine resected specimens to determine the presence of concomitant PEN and IPMN.

Previous studies have discussed the tumorigenesis of concomitant PEN and IPMN. There are two major hypotheses: 1) one cell type in a unique tumoral process could transdifferentiate into another cell type; and 2) two cell types could arise from a common neoplastic progenitor. The former hypothesis is supported by many investigators. For example, Goh et al. noted that (i) the mean age of concomitant patients (60 years) corresponded approximately with that of IPMN, while PEN occurs in much younger patients, (ii) the dominant neoplasm was IPMN in most of the patients, and (iii) diagnosed PENs were of a non-functional nature in most of the patients, based on published reports, which suggests that the PEN component typically arises through transdifferentiation from IPMN [5]. Terada et al. found that argentaffin-, serotonin- and gastrin-secreting cells were present in IPMN but not in normal pancreatic ductal cells, and they suggested that IPMN has the potential for endocrine differentiation [13]. Hashimoto et al. found positivity for exocrine markers expressed on some endocrine tumor cells in a case of mixed PEN and IPMN, and it was noted that the endocrine tumor cells might transdifferentiate to ductal tumor cells [7]. All the features above may be suggestive of tumorigenesis, but is little better than speculation. The present case was a 74-year-old man, who had IPMN dominancy and showed positive glucagon staining in the PEN; however, this case did not provide definitive findings regarding tumorigenesis. We recognize that it is very difficult to assess the tumorigenesis of concomitant PEN or IPMN. It is important to not only assess patients for concomitant PEN and IPMN but also to describe the features with respect to tumorigenesis.

In a search of the PubMed database, we found 19 cases with concomitant PEN and IPMN in eight articles (Table 1). Together with our present case, there are 20 cases with concomitant PEN and IPMN (7 males and 13 females, mean age was 63.7 years old) described in the literature. The locations of PEN and IPMN were not described in nine cases, apparently distant in five cases (case no. 2, 3, 4, 5 and 7 in Table 1), and very close or mixed in the same tumor in six cases (case no. 1, 6, 11, 12, 13 and the present case in Table 1). The preoperative diagnosis was IPMN in 10 cases, PEN in 2 cases, not described in 2 cases, and concomitant PEN and IPMN in only 6 cases. In 6 of the 10 cases whose preoperative diagnosis was IPMN, the size of the PEN was less than $10 \mathrm{~mm}$. The pathological features of PEN were benign in 12 cases, potentially malignant in 3 cases, neuroendocrine carcinoma in 4 cases, and not described in 1 case. The mean tumor sizes (maximum and minimum) of PEN depending on the pathological features were 9.2 (18, 2) $\mathrm{mm}$ in benign, $22.6(28,20) \mathrm{mm}$ in potentially malignant, and $26.5(35,16) \mathrm{mm}$ in neuroendocrine carcinoma. Based on these results from a review of the published literature, concomitant PEN and IPMN is frequently diagnosed as IPMN only and concomitant PEN goes undiagnosed due to its small size. The tumor size of PEN may serve as a guide for clinicopathological features irrespective of the existence of concomitant IPMN [14]. Although post-operative courses are not fully described in most cases, the prognoses for cases of neuroendocrine carcinoma seem pessimistic.

It is important to determine whether the postoperative course and ideal management of cases of concomitant PEN and IPMN differ from that of cases of PEN only or IPMN only. No definitive guidelines have been established in previous case reports of concomitant PEN and IPMN due to the small number of reported cases. Since there have been no reports of cases with concomitant PEN and IPMN in which both PEN and IPMN showed benign or low-grade malignancy, but one or the other showed oncologically aggressive behavior, we believe that there are no synergistic effects between PEN and IPMN, and that cases of concomitant PEN and IPMN should be managed according to their respective natural histories. At the present time, this suggestion is speculative and needs to be validated based on a larger number of cases in the future.

\section{Conclusion}

In conclusion, we encountered a case of concomitant PEN and IPMN. There have been some reports suggesting that the occurrence of concomitant PEN and IPMN has been underreported or undetected because of lack of awareness of the potential for concomitance and poor examination of specimens. It is important to recognize the concomitant neoplasms and determine the actual incidence of this occurrence. We hope that this case presentation will serve as a stimulus for further studies to identify concomitant PEN/IPMN and to better understand the underlying mechanisms.

\section{Consent}

Written informed consent was obtained from the patient for publication of the Case report and accompanying images. A copy of the written consent is available for review by the Editor-in-Chief of this journal.

\section{Abbreviations \\ BPD: Branch pancreas ducts; CA19-9: Carbohydrate antigen 19-9; CEA: Carcinoembryonic antigen; dMPD: Distal section of the main pancreas duct; IPMN: Intraductal papillary mucinous neoplasm; MPD: Main pancreas duct; PEN: Pancreatic endocrine neoplasm; PMPD: Proximal section of the main pancreas duct; PpPD: Pylorus-preserving pancreatoduodenectomy; WHO: World Health Organization.}

\section{Competing interests}

The authors declare that they have no competing interests.

\section{Author's contributions}

YK wrote the paper. MS participated in the operation and supervised the writing of the paper. MT conducted the operation and supervised the writing of the paper. YM, HT and MSakamaoto prepared the pathological 
findings. AU and AT prepared the radiological images. GO and RN participated in the writing of the paper. MTanaka and KM participated in the operation. MK, OI, SK and KA supervised the writing of the paper. YKitagawa represents our surgical department and supervised the writing of the paper. All authors read and approved the final manuscript.

\section{Author details}

'Department of Surgery, School of Medicine, 35 Shinanomachi, Shinjuku, Tokyo 160-8582, Japan. ${ }^{2}$ Department of Pathology, School of Medicine, 35 Shinanomachi, Shinjuku, Tokyo 160-8582, Japan. ${ }^{3}$ Department of Diagnostic Radiology Keio University, School of Medicine, 35 Shinanomachi, Shinjuku, Tokyo 160-8582, Japan. ${ }^{4}$ Department of Surgery, Kawasaki Municipal Hospital, 12-1 Shinkawadori, Kawasaki-shi, Kawasaki-ku, Kanagawa-ken 210-0013, Japan.

Received: 13 August 2012 Accepted: 14 January 2013

Published: 21 March 2013

\section{References}

1. Gumbs AA, Moore PS, Falconi M, Bassi C, Beghelli S, Modlin I, Scarpa A: Review of the clinical, histological, and molecular aspects of pancreatic endocrine neoplasms. J Surg Oncol 2002, 81:45-53.

2. Biankin AV, Biankin SA, Kench JG, Morey AL, Lee CS, Head DR, Eckstein RP, Hugh TB, Henshall SM, Sutherland RL: Aberrant p16 (INK4A) and DPC4/ Smad4 expression in intraductal papillary mucinous tumours of the pancreas is associated with invasive ductal adenocarcinoma. Gut 2002, $50: 861-868$

3. Zhao X, Stabile BE, Mo J, Wang J, French SW: Nesidioblastosis coexisting with islet cell tumor and intraductal papillary mucinous hyperplasia. Arch Pathol Lab Med 2001, 125:1344-1347.

4. Marrache F, Cazals-Hatem D, Kianmanesh R, Palazzo L, Couvelard A, O'Toole D, Maire F, Hammel P, Levy P, Sauvanet A, Ruszniewski P: Endocrine tumor and intraductal papillary mucinous neoplasm of the pancreas: a fortuitous association? Pancreas 2005, 31:79-83.

5. Goh BK, Ooi LL, Kumarasinghe MP, Tan YM, Cheow PC, Chow PK, Chung YF, Wong WK: Clinicopathological features of patients with concomitant intraductal papillary mucinous neoplasm of the pancreas and pancreatic endocrine neoplasm. Pancreatology 2006, 6:520-526.

6. Stukavec J, Jirasek T, Mandys V, Denemark L, Havluj L, Sosna B, Kosmahl M, Zadorova Z: Poorly differentiated endocrine carcinoma and intraductal papillary-mucinous neoplasm of the pancreas: Description of an unusual case. Pathol Res Pract 2007, 203:879-884.

7. Hashimoto $Y$, Murakami $Y$, Uemura $K$, Hayashidani $Y$, Sudo $T$, Ohge $H$, Sueda T, Shimamoto F, Hiyama E: Mixed ductal-endocrine carcinoma derived from intraductal papillary mucinous neoplasm (IPMN) of the pancreas identified by human telomerase reverse transcriptase (hTERT) expression. J Surg Oncol 2008, 97:469-475.

8. Mortelé KJ, Peters HE, Odze RD, Glickman JN, Jajoo K, Banks PA: An unusual mixed tumor of the pancreas: sonographic and MDCT features. JOP 2009, 10:204-208.

9. Larghi A, Stobinski M, Galasso D, Lecca PG, Costamagna G: Concomitant intraductal papillary mucinous neoplasm and pancreatic endocrine tumour: report of two cases and review of the literature. Dig Liver Dis 2009, 41:759-761.

10. Gill KR, Scimeca D, Stauffer J, Krishna M, Woodward TA, Jamil LH, Wallace $\mathrm{MB}$, Nguyen JH, Raimondo M: Pancreatic neuroendocrine tumors among patients with intraductal papillary mucinous neoplasms: real association or just a coincidence? JOP 2009, 10:515-517.

11. Solcia E, Klöppel G, Sobin LH, Williams ED: Histological typing of endocrine tumours. In WHO International Histological Classification of Tumours (2nd edition). Berlin: Springer; 2000

12. Klimstra DS, Arnold R, Capella C, Hruban RH, Klöppel G, Komminoth P, Solcia E, Rindi G: Neuroendocrine neoplasms of the pancreas. In WHO Classification of Tumours of the Digestive System (4th edition). Edited by Bosman FT, Carneiro F, Hruban RH, Theise ND. Lyon, France: IARC Press; 2010:322-326.

13. Terada T, Ohta T, Kitamura Y, Ashida K, Matsunaga Y, Kato M: Endocrine cells in intraductal papillary-mucinous neoplasms of the pancreas. A histochemical and immunohistochemical study. Virchows Arch 1997, 431:31-36.
14. Scarpa A, Mantovani W, Capelli P, Beghelli S, Boninsegna L, Bettini R, Panzuto F, Pederzoli P, delle Fave G, Falconi M: Pancreatic endocrine tumors: improved TNM staging and histopathological grading permit a clinically efficient prognostic stratification of patients. Mod Pathol 2010, 23:824-833.

15. Longnecker DS, Adler G, Hruban RH, Klöppel G: Intraductal papillarymucinous neoplasms of the pancreas. In WHO Classification of Tumours. Pathology and Genetics of Tumours of the Digestive System. Edited by Hamilton SR, Aaltonen LA. Lyon, France: IARC Press; 2000:237-240.

16. Adsay NV, Fukushima N, Furukawa T, Hruban RH, Klimstra DS, Klöppel G, Offerhaus GJA, Pitman MB, Shimizu M, Zamboni G: Intraductal neoplasms of the pancreas. In WHO Classification of Tumours of the Digestive System (4th edition). Edited by Bosman FT, Carneiro F, Hruban RH, Theise ND. Lyon, France: IARC Press; 2010:304-313.

doi:10.1186/1477-7819-11-75

Cite this article as: Kadota et al:: Concomitant pancreatic endocrine neoplasm and intraductal papillary mucinous neoplasm: a case report and literature review. World Journal of Surgical Oncology 2013 11:75.

\section{Submit your next manuscript to BioMed Central and take full advantage of:}

- Convenient online submission

- Thorough peer review

- No space constraints or color figure charges

- Immediate publication on acceptance

- Inclusion in PubMed, CAS, Scopus and Google Scholar

- Research which is freely available for redistribution 